\title{
Molecular testing in the diagnosis of differentiated thyroid carcinomas
}

\author{
Silvia Martina Ferrari ${ }^{1}$, Poupak Fallahi ${ }^{1}$, Ilaria Ruffilli ${ }^{1}$, Giusy Elia ${ }^{1}$, Francesca Ragusa ${ }^{1}$, Sabrina Rosaria \\ Paparo $^{1}$, Salvatore Ulisse ${ }^{2}$, Enke Baldini ${ }^{2}$, Riccardo Giannini ${ }^{3}$, Paolo Miccoli ${ }^{3}$, Alessandro Antonelli ${ }^{1}$, \\ Fulvio Basolo ${ }^{3}$
}

${ }^{1}$ Department of Clinical and Experimental Medicine, University of Pisa, Pisa, Italy; ${ }^{2}$ Department of Surgical Sciences, 'Sapienza' University of Rome, Rome, Italy; ${ }^{3}$ Department of Surgical, Medical, Molecular Pathology and Critical Area, University of Pisa, Pisa, Italy

Contributions: (I) Conception and design: SM Ferrari, P Fallahi, S Ulisse, P Miccoli, A Antonelli, F Basolo; (II) Administrative support: None; (III) Provision of study material or patients: None; (IV) Collection and assembly of data: I Ruffilli, G Elia, F Ragusa, SR Paparo, E Baldini, R Giannini; (V) Data analysis and interpretation: SM Ferrari, P Fallahi, S Ulisse, P Miccoli, A Antonelli, F Basolo; (VI) Manuscript writing: All authors; (VII) Final approval of manuscript: All authors.

Correspondence to: Alessandro Antonelli, MD. Director, Immuno-Endocrine Section of Internal Medicine; Professor of Medicine; Head, Laboratory of Primary Human Cells; Department of Clinical and Experimental Medicine, University of Pisa, School of Medicine, Via Savi, 10, I-56126, Pisa, Italy. Email: alessandro.antonelli@med.unipi.it.

\begin{abstract}
Different genetic mutations and other molecular alterations in papillary thyroid cancer (PTC) and follicular thyroid cancer (FTC) can be detected in fine-needle aspiration (FNA) of thyroid nodules, and can be used successfully to ameliorate cancer diagnosis and management of patients with thyroid nodules. The greatest experience has been obtained with the diagnostic use of BRAF mutation that is strongly specific for malignancy when detected using well-validated techniques. The strongest diagnostic result can be obtained testing FNA samples for a panel of mutations that typically involve TERT, BRAF, PAX8/PPAR $\gamma$, RAS, and RET/PTC. Finding any of these mutations in a thyroid nodule provides strong indication for malignancy and helps to refine clinical management for a significant proportion of patients with indeterminate cytology. The use of molecular markers, as TERT, BRAF, PAX8/PPAR $\gamma$, RAS, and RET/PTC, may be considered for patients with indeterminate FNA cytology (FNAC) to help guide management. In patients with indeterminate TIR3 FNA, the combination of precise molecular marker expression analysis with molecular mutations evaluations could ameliorate significantly the accuracy of cancer diagnosis. However other prospective studies are needed to identify more accurate molecular markers. Finally, the knowledge of these molecular pathways has permitted the development of new targeted therapies for aggressive TC.
\end{abstract}

Keywords: Fine-needle aspiration cytology (FNAC); follicular thyroid cancer (FTC); indeterminate thyroid nodules; molecular diagnostic; papillary thyroid cancer (PTC)

Submitted Sep 08, 2017. Accepted for publication Nov 03, 2017.

doi: $10.21037 /$ gs.2017.11.07

View this article at: http://dx.doi.org/10.21037/gs.2017.11.07

\section{Introduction}

In the United States, thyroid cancer (TC) has the highest rate of growth among diagnosed tumors (1). TC accounts for $6 \%$ of women cancers and less than $3 \%$ of men cancers.

Even if most of TCs are sporadic (2), the nuclear disasters and the resulting radiation exposure (for instance,
Chernobyl, 1986), represent significant risk factors for TC development (3-5).

Papillary TCs (PTCs) and follicular TCs (FTCs) arise from follicular cells and represent the majority of TCs (90\%), while medullary TCs arise from para-follicular C-cells, and are by far less common (5\%). Thyroid malignancies span from the well-differentiated to the poorly 
differentiated or undifferentiated (anaplastic) cancers (6).

Standard treatment for TC usually includes primary surgery (near-total or total thyroidectomy and lymph nodes dissection if necessary), ablation of the thyroid remnant with radioactive iodine (RAI) (based on the tumor stage) and thyroid-stimulating hormone (TSH) suppressive therapy (7).

Follow-up consists of neck ultrasonography (US), performed basally and after TSH-stimulated thyroglobulin assay (7-10).

Though TC has generally a good prognosis, $10 \%$ to $15 \%$ of patients with TC have recurrent disease, and about $5 \%$ will develop metastatic disease not responsive to RAI, and eventually will die from this disease (11-14).

For these reasons an early diagnosis of TC is needed in patients with thyroid nodules. US along with fineneedle aspiration (FNA) cytology (FNAC) are determinant to differentiate benign thyroid nodules from malignant nodules (7).

In order to improve FNAC accuracy in detecting malignancies, testing for oncogene mutations has been proposed $(15,16)$, suggesting that it improves the performance of FNA diagnosis when it is performed in indeterminate cytology, supporting the clinician's decision making process in several settings of uncertain diagnosis. Testing for multiple mutations [BRAF, RAS, RET/PTC, PAX8/peroxisome proliferator-activated receptor (PPAR) $\gamma]$ improves the performance and increases the specificity, but it does not increase the sensitivity as well (15).

For these reasons, molecular diagnostic is recommended in the 2015 revised American Thyroid Association management guidelines in the cases of patients with thyroid nodules and differentiated TC (DTC) with indeterminate cytology (17).

This study aims to review the potential advantages of assessing the gene-related cancer status on samples obtained with FNA, and their prognostic relevance.

\section{Molecular pathways implicated in TC}

\section{Rearranged during transfection (RET)}

RET is a proto-oncogene (located on 10q11.2) encoding a transmembrane protein harboring intracellularly a tyrosine kinase (TK). Activating RET mutations and rearrangements have been reported in human cancers (18). RET/PTC rearrangements have been identified in about $40 \%$ of adult sporadic PTC (19). The most common are RET/PTC1, and RET/PTC3, often reported in microcarcinomas and benign thyroid lesions, leading to hypothesize that RET/ PTC can be determinant for tumor initiation, but not progression (20-24).

\section{RAF kinase}

The BRAF kinase belongs to RAF family proteins; a BRAF mutation [substitution from valine to glutamate at residue $600(\mathrm{~V} 600 \mathrm{E})$ ] is present in approximately $45 \%$ of PTC, associated with tumor aggressiveness (24).

\section{Vascular endothelial growth factor (VEGF)}

TC aggressiveness and increased angiogenesis are correlated (25), and the expression of VEGF/VEGF receptor (VEGFR), platelet-derived growth-factor (PDGF)/ PDGFR, fibroblast growth factor (FGF)/FGFR, epidermal growth factor (EGF)/EGFR, and hepatocyte growth factor (HGF)/c-Met (that stimulate or inhibit angiogenesis) is associated with clinical features of TC (26). VEGF and its principal receptor VEGFR-2 are overexpressed in DTC (27) and are implicated in tumor progression and aggressive behavior. Drugs that target VEGF pathway are indeed a therapeutic approach in TC (28).

\section{Histone acetylation}

On histones the acetylation of NH2-terminal lysine controls cellular differentiation and biological behavior of neoplastic cells.

A more open chromatin configuration leads to an increase in gene transcription rate while a closed chromosomal configuration is linked to transcriptional repression (29).

The dysregulation of histone acetyltransferase or histone deacetylase (HDAC) activity is present in some neoplastic cells (30-33).

The HDAC inhibitors depsipeptide, vorinostat, belinostat, valproic acid, and panobinostat have an antineoplastic effect on TC cells (34).

\section{Epidermal growth factor receptor (EGFR)}

The EGFR cell-surface protein (ErbB-1; HER1 in humans) is part of the ErbB family of receptors (35), a subfamily of four closely related receptor TKs: EGFR (ErbB-1), HER2/ c-neu (ErbB-2), HER3 (ErbB-3) and HER4 (ErbB-4).

Mutations can cause EGFR upregulation or over 
activity, that have been correlated with various cancers, as lung cancer, anal cancer (36) and glioblastoma multiform (in which EGFRvIII results to be the most frequent mutation) (37).

Mutations, amplifications or misregulations of EGFR or of other family members are present in approximately $30 \%$ of all epithelial tumors, and EGFR is determinant in tumor progression and invasion in TC and is overexpressed in anaplastic TC (ATC) (38-40).

\section{$R A S$}

The RAS gene family members N-RAS, H-RAS, and K-RAS encode for intracellular G-proteins participating in the activation of intracellular signal pathways. Approximately $40-50 \%$ of FTCs and $10 \%$ of PTCs show RAS mutations (associated with a more aggressive TC behavior) (41).

\section{TERT}

The major part of normal cells does not have telomerase activity, while the upregulation of TERT transcription/ activity is shown in $80-90 \%$ of malignant tumors. In different kinds of cancer, the presence of TERT promoter mutations, TERT mRNA expression and clinicopathological features are associated, even if the link between the occurrence of TERT promoter mutations and tumor aggressiveness is not known. Even the association between TERT promoter mutations and metastisation/ survival has been reported in different cancers. In follicular cell-derived TC, such mutations are associated with worse prognostic features (age of patients, tumor size and stage) and distant metastases, less response to treatment and poor survival (42-44).

\section{PAX8/peroxisome proliferator-activated receptor $\gamma(P P A R \gamma)$ rearrangements}

PAX8/PPAR $\gamma$ rearrangements are detected in typical FTC (30-40\%) and in Hürthle cell tumors (5\%) (45), and their presence is linked to a good prognosis.

Tumors presenting PAX8/PPAR $\gamma$ rearrangements do not have any RAS mutation, leading to hypothesize the coexistence of two separate mechanisms (PAX8/PPAR $\gamma$ translocation or RAS oncogenic activation) that contribute to the development of FTC (45).

PAX8/PPAR $\gamma$ rearrangements are detected in follicular adenomas (FAs; $2-10 \%$ ), as in the follicular variant of PTC
(FVPTC) $(46,47)$. Approximately $0-1 \%$ of PTCs have PAX8/PPAR $\gamma$ translocations (46).

\section{Molecular diagnosis of thyroid nodules}

Thyroid nodules are very common, since $5 \%$ of women and $1 \%$ of men have palpable nodules in iodine-sufficient countries. However, $19-67 \%$ of randomly selected individuals have thyroid nodules detectable by US (7). TC occurs in $5-15 \%$ of thyroid nodules, depending on age, sex, radiation exposure history, family history, and other factors (48). Early diagnosis of malignant thyroid lesions allows to achieve a curative total thyroidectomy. For this reason, it is crucial to distinguish them among thyroid nodules, which are very often benign (49).

At present the combination of FNA with cytological evaluation is the common preoperative diagnostic method in TC, even though a number between $10 \%$ and $40 \%$ of cytological specimens still result indeterminate for malignancy (50).

Since 2008, the indeterminate cytology is classified in the following three subclasses: "follicular lesion of undetermined significance", "follicular or oncocytic (Hürthle cell) neoplasm", and "suspicious for malignancy". These subclasses express the malignancy chance of $5-10 \%$, $15-30 \%$, and $50-75 \%$, respectively $(51)$.

Molecular testing of FNA biopsies (FNABs), overall for BRAF, and for other markers as RET/PTC, PAX8/ PPAR $\gamma, \mathrm{BRAF}$, and RAS, is possible and can significantly ameliorate the accuracy of the preoperative FNA diagnosis by cytology (52-62). In patient with indeterminate cytology, the investigation for genetic markers (PAX8/PPAR $\gamma, \mathrm{BRAF}$, RAS, and RET/PTC) and protein markers (galectin-3) has demonstrated to improve the accuracy of pre-surgery diagnosis (56-58,63-65). Furthermore, the use of molecular markers (i.e., RET/PTC, BRAF, RAS, PAX8/PPAR $\gamma$ or galectin-3) is now formally advised for indeterminate cytological samples in the "2009 Revised American Thyroid Association (ATA) Management Guidelines for Patients with Thyroid Nodules and Differentiated Thyroid Cancer" (Recommendation rating: C) (7).

\section{$B R A F$}

A number of studies (56-60,64,66-72) have reported that molecular testing for BRAF V600E in thyroid FNA samples significantly ameliorates the accuracy of cytological diagnosis of thyroid nodules. A recent meta-analysis of 
18 studies reporting the results of BRAF testing in 2,766 thyroid FNA samples demonstrated that among 581 BRAFpositive cases, 580 were PTCs (73). Therefore just one BRAF-positive specimen collected from a thyroid nodule of resected gland, disclosed benign nature (74). Even if this case is recognized as a false-negative, the rate of malignancy in BRAF-positive nodules tested by FNA was $99.8 \%$. Several studies have reported that $15-39 \%$ of BRAFpositive FNA samples were indeterminate or non-diagnostic by cytology, demonstrating that testing for BRAF is helpful in establishing a definitive diagnosis of cancer in nodules with indeterminate cytology $(58,60,66,75-77)$.

Many authors have investigated the role of BRAF mutation in PTC; the data indicate that BRAF V600E mutation is an important prognostic molecular marker that improves FNAB diagnostic accuracy, but is complementary to cytology and US (61).

In fact, BRAF V600E mutation was significantly associated with malignant US features (solid composition, marked hypoechogenicity, irregular margin, taller-than-wide shape and the presence of microcalcification) (78), and also with poor prognostic factors and increased tumor size (79).

As regards FTC, detection of BRAF mutation had limited value, but in case of suspicious for malignancy FNABs can be helpful and results are associated with risk of extra-thyroidal extension and metastases after surgery (80).

A perspective study published in 2012 assessed the usefulness of US-guided FNAB in the diagnosis of nodules with/without clinical/US features suggestive for malignancy and to evaluate the contribution of BRAF V600E mutation analysis in the detection of DTC. Thyroid cytoaspirates from 2,421 nodules at least $4 \mathrm{~mm}$ in diameter were done in 1,856 patients submitted to cytological evaluation and bimolecular analysis.

Cytology had high positive predictive value and specificity for the diagnosis of malignant lesions. BRAF V600E mutation was reported in 115 samples, and 80 of them had also a diagnosis of PTC. The evaluation of the presence of a BRAF mutation significantly improved the diagnostic value of cytology, increasing FNAB diagnostic sensitivity for malignant nodules of about $28 \%$ (81).

Recently Howell et al. published a single-center, retrospective review (82) of all patients who had initial thyroidectomy for histologic PTC during 2010. The possible correlation between central compartment lymph node metastasis (CLNM) and available preoperative clinical parameters (as age, gender, tumor size, and BRAF mutation status) was evaluated. Considering the common clinical preoperatively available parameters, the BRAF V600E mutation was the only independent predictor of CLNM in PTC and could be utilized to guide the extent of initial surgery (82).

In a recent paper, Kabaker et al. have confirmed that BRAF-positivity is correlated with the most known suspicious US findings, such as hypoechogenicity, illdefined margins, calcifications, absent halo and tallerthan-wide shape (83). Moreover, the positive predictive value raised to $82 \%$ when three or more US such features were present, while the negative predictive value (BRAFnegativity plus no US features) was $88 \%$ (83).

As up-to-date meta-analysis showed, BRAF mutation offers very high grade of specificity $(97.9 \%)$ and positive predictive value (99.9\%) with only 7 false positives described by 3 studies $(74,84,85)$ of a total of 26 , which included the evaluation of 2,800 malignant and benign thyroid neoplasms. Various techniques have been adopted to detect this marker without affecting the results, which remained constant (61-66,73-86) [from Rodrigues et al. (87)].

\section{RET/PTC}

Testing for RET/PTC rearrangements can be helpful for the diagnosis of TC. In thyroid FNA samples, RET/ PTC detection can ameliorate the preoperative diagnosis of thyroid nodules, especially in samples resulting indeterminate by cytology or without a sufficient amount of cells for the cytological evaluation $(54,60,66)$. In 2 prospective studies $(58,64)$, all 16 thyroid nodules positive for RET/PTC were found to be PTCs after surgery, and many of those nodules were indeterminate by cytology. In another recent study (56) 5/6 nodules positive for RET/ PTC in the FNA material were malignant, and 1 nodule was found to be benign after surgery.

Recently, Ferraz et al. have shown the feasibility of testing for RET/PTC rearrangements from routine FNA (88).

The conclusions of a more recent meta-analysis (87) are that the RET/PTC has shown average values of specificity $(18 \%)$ and positive predictive value (87\%).

\section{$R A S$}

The importance of RAS mutation detection lies in the fact that it represents a marker of FVPTC, which is most difficult to diagnose, in particular by FNAC. RAS mutations are the second most common mutation type detected in consecutive FNA samples from thyroid nodules (89). 
A retrospective study on 341 patients with TC showed an association between malignancy and N-RAS mutation, as well as with tissue inhibitor of metalloproteinases- 1 and FNAC result (90). In several prospective studies $(58,64)$, RAS mutation had a $74 \%$ to $88 \%$ positive predictive value for malignancy. Importantly, RAS mutations were identified in tumors that were difficult to diagnose with only cytology, which is FVPTC and FTC. Some RAS-positive nodules were diagnosed as benign FAs, formally contributing to a false-positive rate. It is likely that RAS-positive FAs serve as precursor lesions for RAS-positive FTC and possibly FVPTC, and RAS mutation apparently predisposes the well-differentiated cancer to dedifferentiation and more aggressive behavior (91-96).

In a more recent meta-analysis (87), RAS mutations have displayed average values of specificity $(23 \%)$ and positive predictive value ( $82 \%)$.

\section{PAX8/PPAR $\gamma$}

Detection of PAX8/PPAR $\gamma$ rearrangement in a follicular lesion is not fully diagnostic for malignancy by itself, but it should encourage the pathologist to perform a complete search for vascular or capsular invasion. Although it may not be seen at first, the invasion is found in many PAX8/ PPAR $\gamma$-positive follicular tumors after examination of the entire capsule in multiple histologic levels $(97,98)$.

PAX8/PPAR $\gamma$ rearrangement can be detected in thyroid FNA samples, and this correlates with the presence of malignancy, even if only few positive cases were reported so far in prospective studies $(58,59)$.

Recently, Ferraz et al. have shown also the feasibility of testing for PAX8/PPAR $\gamma$ rearrangements from routine FNA (88).

In a more recent meta-analysis (87), PAX8/PPAR $\gamma$ mutations have shown average values of specificity (20\%) and positive predictive value (100\%).

\section{Multiple testing}

One prospective study, which compared cytology alone or combined with molecular testing for RET/PTC, BRAF, PAX8/PPAR $\gamma$, and RAS mutations, demonstrated to increase the FNA sensitivity for malignancy of thyroid nodules from $44 \%$ to $80 \%$, respectively (58).

After surgery, 31/32 (97\%) mutation-positive nodules were diagnosed as malignant, and $1 / 32(3 \%)$ as a RASpositive $\mathrm{FA}$, suggesting the detection of each mutation can be considered a good predictor of malignancy (58). After surgery, considering the indeterminate FNA samples, molecular testing permitted to identify $15 / 21$ (71\%) malignant nodules, indicating that molecular testing can ameliorate the diagnosis of indeterminate FNAs (58). Another paper showed that the sensitivity of malignant diagnosis in FNA rised from $60 \%$ to $90 \%$, combining cytology with molecular testing for TRK, PPAR $\gamma$, BRAF, RAS, and RET mutations to cytology alone (64). Mutations were associated with cancers in 61/67 mutation-positive patients (91\%) and with benign $\mathrm{FA}$ in $6 / 67$ mutationpositive cases $(9 \%)$, indicating as the previous study that the presence of a mutation could be a good predictor of malignancy. The usefulness of these markers in the presurgery setting is still under investigation.

Recently in a prospective study of Borrelli et al. (99) different consecutive FNA samples were assessed by independent cytological and molecular means. BRAF was mutated (by Sanger method) in the suspicious and malignant cases, but not in TIR1, TIR2, and TIR3 cases, suggesting that BRAF mutational analysis does not improve the cytological diagnosis.

A BRAF mutation was reported in only 3 patients (4.4\%), analyzing the indeterminate nodules (cytological TIR3 cases) by the Easy BRAF kit, that is a very sensitive realtime-based method.

The above reported data indicate that the frequency of BRAF mutations in indeterminate cases was low also using a very sensitive method, depending on the expertise of the cytopathologist, on the accuracy to classify the TIR3 class, and on a selection bias due to the geographic region where the evaluation of the patients was conducted.

An attentive expression profiling analysis was performed to identify new potential diagnostic markers.

Thirty/68 cases of indeterminate nodules were submitted to surgery upon a clinical evaluation, and in $16 / 30$ a histological diagnosis of benign lesions was done, and in 14 of malignant lesions. The malignancy rate in these 30 indeterminate FNA patients was high probably because the first group of patients undergoing surgery showed suspicious aspects with neck ultrasound (i.e., $>8 \mathrm{~mm}$; a hypoechogenic, solid appearance; irregular or blurred margins; presence of microcalcification).

RT Profiler PCR arrays (100) were used for expression profiling analysis in all the 30 patients submitted to surgery. This technique permits to evaluate the expression profile of a specific panel of genes, and was used to analyze 84 genes participating in cell-matrix interactions, cell-cell adhesion, 
and ECM remodeling. In tumors, between epithelial and microenvironment stroma cells, dynamic and reciprocal interactions, involving CAMs (i.e., integrin and CD44), ECM non-cellular components (i.e., thrombospondin 1 and FN1), and soluble cytokines exist. Furthermore, in human tumors the composition of CAMs and ECMs is changed and affects cell growth, survival, migration, adhesion, and other stromal and neoplastic cells features (101-103).

The following genes were identified: 6 genes upregulated in malignant nodules with respect to benign ones ["transforming growth factor, $\beta$-induced (TGFBI), cadherin 1 , type 1 (CDH1), collagen, type I, $\alpha 1$ (COL1A1), catenin (cadherin-associated protein), $\alpha 1,102 \mathrm{kDa}$ (CTNNA1), integrin $\alpha 3$ (ITGA3), and fibronectin 1 (FN1)"]; 6 genes strongly upregulated in FVPTCs vs. FAs ["integrin $\beta 2$ (ITGB2), FN1, connective tissue growth factor (CTGF), laminin $\gamma 1$ (LAMC1), catenin (cadherin-associated protein), $\delta 1$ (CTNND1), and integrin $\alpha \mathrm{V}$ (ITGAV)"].

Moreover, 6 deregulated genes, $\{4$ upregulated genes [secreted phosphoprotein 1 (SPP1), CTNNA1, TIMP metallopeptidase inhibitor 1 (TIMP1), and FN1] and 2 downregulated genes (ADAMTS1 and SELL)\}, were reported in BRAF-mutated PTCs vs. wild type tumors.

These data agree with previously reported differences between malignant and benign thyroid nodules for molecules implicated in cell-cell adhesion, cytoskeletal reorganization, and cell matrix interactions, such as many collagens, catenin, FN1, or integrin (103-110).

\section{Conclusions}

Several genetic mutations occurring in PTCs and FTCs can be detected in FNA of thyroid nodules, and can be used to ameliorate cancer diagnosis and management of patients with thyroid nodules. The greatest experience has been obtained with the diagnostic use of BRAF mutation, which is strongly specific for malignancy when detected using well-validated techniques. The strongest diagnostic result can be obtained testing FNA samples for a panel of mutations that typically involves BRAF, RAS, TERT, RET/ PTC, and PAX8/PPAR $\gamma$. Finding any of these mutations in a thyroid nodule provides strong indication for malignancy and helps to refine clinical management for a significant proportion of patients with indeterminate cytology.

What we know today about the diagnostic value of molecular markers it is reported in the Revised Management Guidelines for Patients with Thyroid Nodules and Differentiated Thyroid Cancer, recently published by the ATA (7). This statements suggest to consider gene markers such as RET/PTC, BRAF, PAX8/PPAR $\gamma$, RAS and in the pre-operative setting of patient whose cytology has indeterminate result at FNAC.

The use of these and other emerging molecular markers is expected to ameliorate significantly the accuracy of cancer diagnosis in thyroid nodules. However other prospective studies are needed to identify more accurate molecular markers. Finally, the knowledge of these molecular pathways has permitted the development of new targeted therapies for aggressive TC $(111,112)$.

The most controversial area of TC research deals with the distinction of FAs from FVPTCs that are strongly connected cancers, whose differential diagnosis is arduous. Up to now, molecular markers have been used to better characterize follicular-derived TC hoping that some of these markers will be helpful to define indeterminate FNA samples.

Some genes, earlier involved in TC pathogenesis, have been identified as able to differentiate FVPTCs from FAs. The expression of CTGF protein was significantly upregulated in TC tissues with respect to benign thyroid tumors (113) and CTGF mRNA expression was significantly different in FAs or FVPTCs, confirming that CTGF could be implicated in the malignancy of TCs. Moreover, in FVPTCs it was shown an overexpression of ITGAV and ITGB1, belonging to the integrin superfamily, as earlier reported in malignant TC $(114,115)$. It was also shown that ECM protein FN1 mRNA expression was significantly increased in FVPTCs vs. FAs. In 1988 FN1 was demonstrated to be overexpressed in TCs by immunohistochemistry for the first time (116), and in 2004 galectin 3 and oncofetal fibronectin were indicated as potential markers for the preoperative diagnosis of TCs (117). Moreover, it was reported that FN1 expression was more strongly linked to malignancy with respect to adenomas and nonmalignant tissues (106).

Now a similar expression rate by semiquantitative, RTPCR and immunohistochemistry is reported, even if the only statistically significant molecule was ITGB2. It has to be noted that recently FNA has been used to evaluate the chemosensitivity of primary TC cells in humans. This might ameliorate the response to treatment, as in vitro drug screening with human primary TC cells has a $60 \%$ positive predictive and a $90 \%$ negative predictive value of clinical response in vivo in the same patient. This could permit not to administer inactive drugs to patients $(118,119)$.

In conclusion, BRAF mutation analysis alone does 
not permit to ameliorate the diagnostic accuracy. In indeterminate TIR3 FNA patients, the combination of precise molecular marker expression analysis with molecular mutations evaluations could be assessed. For these reasons, a further expression analysis of specific molecular markers is necessary to draw decisive conclusions.

\section{Acknowledgements}

None.

\section{Footnote}

Conflicts of Interest: The authors have no conflicts of interest to declare.

\section{References}

1. Siegel R, Naishadham D, Jemal A. Cancer statistics, 2013. CA Cancer J Clin 2013;63:11-30.

2. Detours V, Wattel S, Venet D, et al. Absence of a specific radiation signature in post-Chernobyl thyroid cancers. $\mathrm{Br}$ J Cancer 2005;92:1545-52.

3. Spinelli C, Bertocchini A, Antonelli A, et al. Surgical therapy of the thyroid papillary carcinoma in children: experience with 56 patients $<$ or $=16$ years old. $\mathrm{J}$ Pediatr Surg 2004;39:1500-5.

4. Antonelli A, Miccoli P, Derzhitski VE, et al. Epidemiologic and clinical evaluation of thyroid cancer in children from the Gomel region (Belarus). World J Surg 1996;20:867-71.

5. Miccoli P, Antonelli A, Spinelli C, et al. Completion total thyroidectomy in children with thyroid cancer secondary to the Chernobyl accident. Arch Surg 1998;133:89-93.

6. Sherman SI. Thyroid carcinoma. Lancet 2003;361:501-11.

7. American Thyroid Association (ATA) Guidelines Taskforce on Thyroid Nodules and Differentiated Thyroid Cancer, Cooper DS, Doherty GM, et al. Revised American Thyroid Association management guidelines for patients with thyroid nodules and differentiated thyroid cancer. Thyroid 2009;19:1167-214.

8. Antonelli A, Miccoli P, Ferdeghini M, et al. Role of neck ultrasonography in the follow-up of patients operated on for thyroid cancer. Thyroid 1995;5:25-8.

9. Antonelli A, Miccoli P, Fallahi P, et al. Role of neck ultrasonography in the follow-up of children operated on for thyroid papillary cancer. Thyroid 2003;13:479-84.

10. Antonelli A, Miccoli P, Fallahi P, et al. Thyrotropinstimulated serum thyroglobulin combined with neck ultrasonography has the highest sensitivity in monitoring differentiated thyroid carcinoma in children. Surgery 2006;140:1035-41; discussion 1041-2.

11. Jemal A, Siegel R, Ward E, et al. Cancer statistics, 2009. CA Cancer J Clin 2009;59:225-49.

12. Robbins J, Merino MJ, Boice JD Jr, et al. Thyroid cancer: a lethal endocrine neoplasm. Ann Intern Med 1991;115:133-47.

13. Gilliland FD, Hunt WC, Morris DM, et al. Prognostic factors for thyroid carcinoma. A population-based study of 15,698 cases from the Surveillance, Epidemiology and End Results (SEER) program 1973-1991. Cancer 1997;79:564-73.

14. Antonelli A, Ferrari SM, Fallahi P, et al. Dysregulation of secretion of CXC alpha-chemokine CXCL10 in papillary thyroid cancer: modulation by peroxisome proliferatoractivated receptor-gamma agonists. Endocr Relat Cancer 2009;16:1299-311.

15. Albarel F, Conte-Devolx B, Oliver C. From nodule to differentiated thyroid carcinoma: contributions of molecular analysis in 2012. Ann Endocrinol (Paris) 2012;73:155-64.

16. Nikiforova MN, Nikiforov YE. Molecular diagnostics and predictors in thyroid cancer. Thyroid 2009;19:1351-61.

17. Haugen BR, Alexander EK, Bible KC, et al. 2015 American thyroid association management guidelines for adult patients with thyroid nodules and differentiated thyroid cancer: the American thyroid association guidelines task force on thyroid nodules and differentiated thyroid cancer. Thyroid 2016;26:1-133.

18. de Groot JW, Links TP, Plukker JT, et al. RET as a diagnostic and therapeutic target in sporadic and hereditary endocrine tumors. Endocr Rev 2006;27:535-60.

19. Nikiforov YE. Thyroid carcinoma: molecular pathways and therapeutic targets. Mod Pathol 2008;21:S37-43.

20. Marotta V, Guerra A, Sapio MR, et al. RET/PTC rearrangement in benign and malignant thyroid diseases: a clinical standpoint. Eur J Endocrinol 2011;165:499-507.

21. Fagin JA. Challenging dogma in thyroid cancer molecular genetics--role of RET/PTC and BRAF in tumor initiation. J Clin Endocrinol Metab 2004;89:4264-6.

22. Santoro M, Melillo RM, Fusco A. RET/PTC activation in papillary thyroid carcinoma: European Journal of Endocrinology Prize Lecture. Eur J Endocrinol 2006;155:645-53.

23. Antonelli A, Fallahi P, Ferrari SM, et al. RET TKI: potential role in thyroid cancers. Curr Oncol Rep 2012;14:97-104.

24. Xing M. BRAF mutation in thyroid cancer. Endocr Relat Cancer 2005;12:245-62. 
25. Marotta V, Franzese MD, Del Prete M, et al. Targeted therapy with kinase inhibitors in aggressive endocrine tumors. Expert Opin Pharmacother 2013;14:1187-203.

26. Tan A, Xia N, Gao F, et al. Angiogenesis-inhibitors for metastatic thyroid cancer. Cochrane Database Syst Rev 2010;3:CD007958.

27. Bunone G, Vigneri P, Mariani L, et al. Expression of angiogenesis stimulators and inhibitors in human thyroid tumors and correlation with clinical pathological features. Am J Pathol 1999;155:1967-76.

28. Cao Y. VEGF-targeted cancer therapeutics-paradoxical effects in endocrine organs. Nat Rev Endocrinol 2014;10:530-9.

29. Minucci S, Pelicci PG. Histone deacetylase inhibitors and the promise of epigenetic (and more) treatments for cancer. Nat Rev Cancer 2006;6:38-51.

30. Bhalla K, List A. Histone deacetylase inhibitors in myelodysplastic syndrome. Best Pract Res Clin Haematol 2004;17:595-611.

31. Mahlknecht U, Hoelzer D. Histone acetylation modifiers in the pathogenesis of malignant disease. Mol Med 2000;6:623-44.

32. Cress WD, Seto E. Histone deacetylases, transcriptional control, and cancer. J Cell Physiol 2000;184:1-16.

33. Marks P, Rifkind RA, Richon VM, et al. Histone deacetylases and cancer: causes and therapies. Nat Rev Cancer 2001;1:194-202.

34. Batty N, Malouf GG, Issa JP. Histone deacetylase inhibitors as anti-neoplastic agents. Cancer Lett 2009;280:192-200.

35. Herbst RS. Review of epidermal growth factor receptor biology. Int J Radiat Oncol Biol Phys 2004;59:21-6.

36. Walker F, Abramowitz L, Benabderrahmane D, et al. Growth factor receptor expression in anal squamous lesions: modifications associated with oncogenic human papillomavirus and human immunodeficiency virus. Hum Pathol 2009;40:1517-27.

37. Kuan CT, Wikstrand CJ, Bigner DD. EGF mutant receptor vIII as a molecular target in cancer therapy. Endocr Relat Cancer 2001;8:83-96.

38. Knauf JA. Does the epidermal growth factor receptor play a role in the progression of thyroid cancer? Thyroid 2011;21:1171-4.

39. Hoffmann S, Burchert A, Wunderlich A, et al. Differential effects of cetuximab and AEE 788 on epidermal growth factor receptor (EGF-R) and vascular endothelial growth factor receptor (VEGF-R) in thyroid cancer cell lines. Endocrine 2007;31:105-13.
40. Yeh MW, Rougier JP, Park JW, et al. Differentiated thyroid cancer cell invasion is regulated through epidermal growth factor receptor-dependent activation of matrix metalloproteinase (MMP)-2/gelatinase A. Endocr Relat Cancer 2006;13:1173-83.

41. Howell GM, Hodak SP, Yip L. RAS mutations in thyroid cancer. Oncologist 2013;18:926-32.

42. Shrestha RT, Karunamurthy A, Amin K, et al. Multiple mutations detected preoperatively may predict aggressive behavior of papillary thyroid cancer and guide management--a case report. Thyroid 2015;25:1375-8.

43. Pestana A, Vinagre J, Sobrinho-Simões M, et al. TERT biology and function in cancer: beyond immortalisation. J Mol Endocrinol 2017;58:R129-46.

44. Liu R, Xing M. TERT promoter mutations in thyroid cancer. Endocr Relat Cancer 2016;23:R143-55.

45. Nikiforova MN, Lynch RA, Biddinger PW, et al. RAS point mutations and PAX8-PPAR gamma rearrangement in thyroid tumors: evidence for distinct molecular pathways in thyroid follicular carcinoma. J Clin Endocrinol Metab 2003;88:2318-26.

46. Marques AR, Espadinha C, Catarino AL, et al. Expression of PAX8-PPAR gamma 1 rearrangements in both follicular thyroid carcinomas and adenomas. J Clin Endocrinol Metab 2002;87:3947-52.

47. Castro P, Rebocho AP, Soares RJ, et al. PAX8PPARgamma rearrangement is frequently detected in the follicular variant of papillary thyroid carcinoma. J Clin Endocrinol Metab 2006;91:213-20.

48. Hegedüs L. Clinical practice. The thyroid nodule. N Engl J Med 2004;351:1764-71.

49. Mahar SA, Husain A, Islam N. Fine needle aspiration cytology of thyroid nodule: diagnostic accuracy and pitfalls. J Ayub Med Coll Abbottabad 2006;18:26-9.

50. Gharib H, Papini E. Thyroid nodules: clinical importance, assessment, and treatment. Endocrinol Metab Clin North Am 2007;36:707-35.

51. Baloch ZW, LiVolsi VA, Asa SL, et al. Diagnostic terminology and morphologic criteria for cytologic diagnosis of thyroid lesions: a synopsis of the National Cancer Institute Thyroid Fine-Needle Aspiration State of the Science Conference. Diagn Cytopathol 2008; 36:425-37.

52. Xing M, Clark D, Guan H, et al. BRAF mutation testing of thyroid fine-needle aspiration biopsy specimens for preoperative risk stratification in papillary thyroid cancer. J Clin Oncol 2009;27:2977-82.

53. Xing M. BRAF mutation in papillary thyroid cancer: pathogenic role, molecular bases, and clinical implications. 
Endocr Rev 2007;28:742-62.

54. Cheung CC, Carydis B, Ezzat S, et al. Analysis of ret/PTC gene rearrangements refines the fine needle aspiration diagnosis of thyroid cancer. J Clin Endocrinol Metab 2001;86:2187-90.

55. Eszlinger M, Paschke R. Molecular fine-needle aspiration biopsy diagnosis of thyroid nodules by tumor specific mutations and gene expression patterns. Mol Cell Endocrinol 2010;322:29-37.

56. Moses W, Weng J, Sansano I, et al. Molecular testing for somatic mutations improves the accuracy of thyroid fineneedle aspiration biopsy. World J Surg 2010;34:2589-94.

57. Musholt TJ, Fottner C, Weber MM, et al. Detection of papillary thyroid carcinoma by analysis of BRAF and RET/PTC1 mutations in fine-needle aspiration biopsies of thyroid nodules. World J Surg 2010;34:2595-603.

58. Nikiforov YE, Steward DL, Robinson-Smith TM, et al. Molecular testing for mutations in improving the fineneedle aspiration diagnosis of thyroid nodules. J Clin Endocrinol Metab 2009;94:2092-8.

59. Ohori NP, Nikiforova MN, Schoedel KE, et al. Contribution of molecular testing to thyroid fine-needle aspiration cytology of "follicular lesion of undetermined significance/atypia of undetermined significance". Cancer Cytopathol 2010;118:17-23.

60. Salvatore G, Giannini R, Faviana P, et al. Analysis of BRAF point mutation and RET/PTC rearrangement refines the fine-needle aspiration diagnosis of papillary thyroid carcinoma. J Clin Endocrinol Metab 2004;89:5175-80.

61. Zatelli MC, Trasforini G, Leoni S, et al. BRAF V600E mutation analysis increases diagnostic accuracy for papillary thyroid carcinoma in fine-needle aspiration biopsies. Eur J Endocrinol 2009;161:467-73.

62. Guo F, Hou P, Shi B. Detection of BRAF mutation on fine needle aspiration biopsy specimens: diagnostic and clinical implications for papillary thyroid cancer. Acta Cytol 2010;54:291-5.

63. Bartolazzi A, Orlandi F, Saggiorato E, et al. Galectin-3expression analysis in the surgical selection of follicular thyroid nodules with indeterminate fine-needle aspiration cytology: a prospective multicentre study. Lancet Oncol 2008;9:543-9.

64. Cantara S, Capezzone M, Marchisotta S, et al. Impact of proto-oncogene mutation detection in cytological specimens from thyroid nodules improves the diagnostic accuracy of cytology. J Clin Endocrinol Metab 2010;95:1365-9.

65. Franco C, Martinez V, Allamand JP, et al. Molecular markers in thyroid fine-needle aspiration biopsy: a prospective study. Appl Immunohistochem Mol Morphol 2009;17:211-5.

66. Pizzolanti G, Russo L, Richiusa P, et al. Fine-needle aspiration molecular analysis for the diagnosis of papillary thyroid carcinoma through BRAF V600E mutation and RET/PTC rearrangement. Thyroid 2007;17:1109-15.

67. Xing M, Tufano RP, Tufaro AP, et al. Detection of BRAF mutation on fine needle aspiration biopsy specimens: a new diagnostic tool for papillary thyroid cancer. J Clin Endocrinol Metab 2004;89:2867-72.

68. Domingues R, Mendonca E, Sobrinho L, et al. Searching for RET/PTC rearrangements and BRAF V599E mutation in thyroid aspirates might contribute to establish a preoperative diagnosis of papillary thyroid carcinoma. Cytopathology 2005;16:27-31.

69. Sapio MR, Guerra A, Posca D, et al. Combined analysis of galectin-3 and BRAFV600E improves the accuracy of fine-needle aspiration biopsy with cytological findings suspicious for papillary thyroid carcinoma. Endocr Relat Cancer 2007;14:1089-97.

70. Sapio MR, Posca D, Raggioli A, et al. Detection of RET/ PTC, TRK and BRAF mutations in preoperative diagnosis of thyroid nodules with indeterminate cytological findings. Clin Endocrinol (Oxf) 2007;66:678-83.

71. Moon HJ, Kwak JY, Kim EK, et al. The role of BRAFV600E mutation and ultrasonography for the surgical management of a thyroid nodule suspicious for papillary thyroid carcinoma on cytology. Ann Surg Oncol 2009;16:3125-31.

72. Marchetti I, Iervasi G, Mazzanti CM, et al. Detection of the BRAF(V600E) mutation in fine needle aspiration cytology of thyroid papillary microcarcinoma cells selected by manual macrodissection: an easy tool to improve the preoperative diagnosis. Thyroid 2012;22:292-8.

73. Nikiforov YE. Molecular diagnostics of thyroid tumors. Arch Pathol Lab Med 2011;135:569-77.

74. Chung KW, Yang SK, Lee GK, et al. Detection of BRAFV600E mutation on fine needle aspiration specimens of thyroid nodule refines cyto-pathology diagnosis, especially in BRAF600E mutation-prevalent area. Clin Endocrinol (Oxf) 2006;65:660-6.

75. Jo YS, Huang S, Kim YJ, et al. Diagnostic value of pyrosequencing for the BRAF V600E mutation in ultrasound-guided fine-needle aspiration biopsy samples of thyroid incidentalomas. Clin Endocrinol (Oxf) 2009;70:139-44.

76. Cohen Y, Rosenbaum E, Clark DP, et al. Mutational 
analysis of BRAF in fine needle aspiration biopsies of the thyroid: a potential application for the preoperative assessment of thyroid nodules. Clin Cancer Res 2004;10:2761-5.

77. Kim SK, Kim DL, Han HS, et al. Pyrosequencing analysis for detection of a BRAFV600E mutation in an FNAB specimen of thyroid nodules. Diagn Mol Pathol 2008;17:118-25.

78. Nam SY, Han BK, Ko EY, et al. BRAF V600E mutation analysis of thyroid nodules needle aspirates in relation to their ultrasongraphic classification: a potential guide for selection of samples for molecular analysis. Thyroid 2010;20:273-9.

79. Lee EJ, Song KH, Kim DL, et al. The BRAF(V600E) mutation is associated with malignant ultrasonographic features in thyroid nodules. Clin Endocrinol (Oxf) 2011;75:844-50.

80. Patel A, Klubo-Gwiezdzinska J, Hoperia V, et al. BRAF(V600E) mutation analysis from May-Grunwald Giemsa-stained cytological samples as an adjunct in identification of high-risk papillary thyroid carcinoma. Endocr Pathol 2011;22:195-9.

81. Rossi M, Buratto M, Bruni S, et al. Role of ultrasonographic/clinical profile, cytology, and BRAF V600E mutation evaluation in thyroid nodule screening for malignancy: a prospective study. J Clin Endocrinol Metab 2012;97:2354-61.

82. Howell GM, Nikiforova MN, Carty SE, et al. BRAF V600E mutation independently predicts central compartment lymph node metastasis in patients with papillary thyroid cancer. Ann Surg Oncol 2013;20:47-52.

83. Kabaker AS, Tublin ME, Nikiforov YE, et al. Suspicious ultrasound characteristics predict BRAF V600E-positive papillary thyroid carcinoma. Thyroid 2012;22:585-9.

84. Kim SK, Hwang TS, Yoo YB, et al. Surgical results of thyroid nodules according to a management guideline based on the BRAF(V600E) mutation status. J Clin Endocrinol Metab 2011;96:658-64.

85. Kim SW, Lee JI, Kim JW, et al. BRAFV600E mutation analysis in fine-needle aspiration cytology specimens for evaluation of thyroid nodule: a large series in a BRAFV600E-prevalent population. J Clin Endocrinol Metab 2010;95:3693-700.

86. Jin L, Sebo TJ, Nakamura N, et al. BRAF mutation analysis in fine needle aspiration (FNA) cytology of the thyroid. Diagn Mol Pathol 2006;15:136-43.

87. Rodrigues HG, de Pontes AA, Adan LF. Use of molecular markers in samples obtained from preoperative aspiration of thyroid. Endocr J 2012;59:417-24.

88. Ferraz C, Rehfeld C, Krogdahl A, et al. Detection of PAX8/PPARG and RET/PTC rearrangements is feasible in routine air-dried fine needle aspiration smears. Thyroid 2012;22:1025-30.

89. Gupta N, Dasyam AK, Carty SE, et al. RAS mutations in thyroid FNA specimens are highly predictive of predominantly low-risk follicular-pattern cancers. J Clin Endocrinol Metab 2013;98:E914-22.

90. Mehta V, Nikiforov YE, Ferris RL. Use of molecular biomarkers in FNA specimens to personalize treatment for thyroid surgery. Head Neck 2013;35:1499-506.

91. Basolo F, Pisaturo F, Pollina LE, et al. N-ras mutation in poorly differentiated thyroid carcinomas: correlation with bone metastases and inverse correlation to thyroglobulin expression. Thyroid 2000;10:19-23.

92. Fagin JA. Minireview: branded from the start-distinct oncogenic initiating events may determine tumor fate in the thyroid. Mol Endocrinol 2002;16:903-11.

93. Garcia-Rostan G, Zhao H, Camp RL, et al. ras mutations are associated with aggressive tumor phenotypes and poor prognosis in thyroid cancer. J Clin Oncol 2003;21:3226-35.

94. Hara H, Fulton N, Yashiro T, et al. N-ras mutation: an independent prognostic factor for aggressiveness of papillary thyroid carcinoma. Surgery 1994;116:1010-6.

95. Saavedra HI, Knauf JA, Shirokawa JM, et al. The RAS oncogene induces genomic instability in thyroid PCCL3 cells via the MAPK pathway. Oncogene 2000;19:3948-54.

96. Zhu Z, Gandhi M, Nikiforova MN, et al. Molecular profile and clinical-pathologic features of the follicular variant of papillary thyroid carcinoma. An unusually high prevalence of ras mutations. Am J Clin Pathol 2003;120:71-7.

97. French CA, Alexander EK, Cibas ES, et al. Genetic and biological subgroups of low-stage follicular thyroid cancer. Am J Pathol 2003;162:1053-60.

98. Nikiforova MN, Biddinger PW, Caudill CM, et al. PAX8PPARgamma rearrangement in thyroid tumors: RT-PCR and immunohistochemical analyses. Am J Surg Pathol 2002;26:1016-23.

99. Borrelli N, Ugolini C, Giannini R, et al. Role of gene expression profiling in defining indeterminate thyroid nodules in addition to BRAF analysis. Cancer Cytopathol 2016;124:340-9.

100. Nikiforov YE, Nikiforova MN. Molecular genetics and diagnosis of thyroid cancer. Nat Rev Endocrinol 2011;7:569-80.

101.Polyak K, Haviv I, Campbell IG. Co-evolution of tumor cells and their microenvironment. Trends Genet 
2009;25:30-8.

102. Albelda SM. Role of integrins and other cell adhesion molecules in tumor progression and metastasis. Lab Invest 1993;68:4-17.

103. Clezardin P. Recent insights into the role of integrins in cancer metastasis. Cell Mol Life Sci 1998;54:541-8.

104. Nakano T, Kusunoki T, Funasaka K, et al. Study of type I and IV collagenase activity in human thyroid diseases. Nihon Jibiinkoka Gakkai Kaiho 1995;98:937-41.

105.Jarzab B, Wiench M, Fujarewicz K, et al. Gene expression profile of papillary thyroid cancer: sources of variability and diagnostic implications. Cancer Res 2005;65:1587-97

106. Prasad ML, Pellegata NS, Huang Y, et al. Galectin-3, fibronectin- 1, CITED-1, HBME1 and cytokeratin-19 immunohistochemistry is useful for the differential diagnosis of thyroid tumors. Mod Pathol 2005;18:48-57.

107.Lubitz CC, Ugras SK, Kazam JJ, et al. Microarray analysis of thyroid nodule fine-needle aspirates accurately classifies benign and malignant lesions. J Mol Diagn 2006;8:490-8.

108. Finley DJ, Zhu B, Barden CB, et al. Discrimination of benign and malignant thyroid nodules by molecular profiling. Ann Surg 2004;240:425-36.

109. Wasenius VM, Hemmer S, Kettunen E, et al. Hepatocyte growth factor receptor, matrix metalloproteinase-11, tissue inhibitor of metalloproteinase-1, and fibronectin are upregulated in papillary thyroid carcinoma: a cDNA and tissue microarray study. Clin Cancer Res 2003;9:68-75.

110. Prasad NB, Somervell H, Tufano RP, et al. Identification of genes differentially expressed in benign versus malignant thyroid tumors. Clin Cancer Res 2008;14:3327-37.

111. Antonelli A, Fallahi P, Ferrari SM, et al. New targeted

Cite this article as: Ferrari SM, Fallahi P, Ruffilli I, Elia G, Ragusa F, Paparo SR, Ulisse S, Baldini E, Giannini R, Miccoli P, Antonelli A, Basolo F. Molecular testing in the diagnosis of differentiated thyroid carcinomas. Gland Surg 2018;7(Suppl 1):S19-S29. doi: 10.21037/gs.2017.11.07 therapies for thyroid cancer. Curr Genomics 2011;12:626-31.

112.Antonelli A, Bocci G, La Motta C, et al. CLM94, a novel cyclic amide with anti-VEGFR-2 and antiangiogenic properties, is active against primary anaplastic thyroid cancer in vitro and in vivo. J Clin Endocrinol Metab 2012;97:E528-36.

113. Wang G, Zhang W, Meng W, et al. Expression and clinical significante of connective tissue growth factor in thyroid carcinomas. J Int Med Res 2013;41:1214-20.

114.Liu X, He M, Hou Y, et al. Expression profiles of microRNAs and their target genes in papillary thyroid carcinoma. Oncol Rep 2013;29:1415-20.

115. Dahlman T, Grimelius L, Wallin G, et al. Integrins in thyroid tissue: upregulation of alpha2beta 1 in anaplastic thyroid carcinoma. Eur J Endocrinol 1998;138:104-12.

116. Sugenoya A, Usuda N, Adachi W, et al. Immunohistochemical studies on the localization of fibronectin in human thyroid neoplastic tissues. Endocrinol Jpn 1988;35:111-20.

117. Giannini R, Faviana P, Cavinato T, et al. Galectin-3 and oncofetal-fibronectin expression in thyroid neoplasia as assessed by reverse transcription-polymerase chain reaction and immunochemistry in cytologic and pathologic specimens. Thyroid 2003;13:765-70.

118. Antonelli A, Ferrari SM, Fallahi P, et al. Thiazolidinediones and antiblastics in primary human anaplastic thyroid cancer cells. Clin Endocrinol (Oxf) 2009;70:946-53.

119. Antonelli A, Ferrari SM, Fallahi P. Primary cell cultures from anaplastic thyroid cancer obtained by fine-needle aspiration used for chemosensitivity tests. Clin Endocrinol (Oxf) 2008;69:148-52. 